\title{
Spin-phonon interaction in transition-metal difluoride antiferromagnets: Theory and experiment
}

\author{
M.G. Cottam \\ Department of Physics and Astronomy, University of Western Ontario, London, Ontario, Canada N6A 3K7 \\ E-mail: cottam@uwo.ca \\ D.J. Lockwood \\ Measurement Science and Standards, National Research Council, Ottawa, Ontario, Canada K1A OR6 \\ E-mail: david.lockwood@nrc-cnrc.gc.ca
}

Received July 26, 2018, published online November 26, 2018

\begin{abstract}
An overall comparative study is made of the spin-phonon interactions in several rutile-structure transitionmetal difluorides, specifically $\mathrm{FeF}_{2}, \mathrm{MnF}_{2}, \mathrm{NiF}_{2}$, and $\mathrm{CoF}_{2}$, in terms of recent developments obtained experimentally using inelastic light scattering spectroscopy and theoretically using a modified mean-field approach to estimate spin-pair correlation functions. New experimental data are presented here and interpreted within an extended and comprehensive theoretical treatment to yield estimates for the spin-phonon coupling coefficients and the relative magnitudes of the magneto-optical coupling coefficients.
\end{abstract}

Keywords: antiferromagnets, spin-phonon coupling, rutile structure, magneto-optical coupling, Raman spectroscopy.

\section{Introduction}

The invention of the laser led to a revolution in the applications of inelastic light scattering spectroscopy. In the 1960s, laser Raman scattering spectroscopy was widely used to study the electronic and magnetic properties of transitionmetal and rare-earth compounds. Many interesting and novel features of the effects of magnetic ordering in such solids were reported [1]. These early studies focused primarily on the fundamental magnetic excitation, the magnon, but also investigated associated excitons at higher energies [2]. Nowadays, Raman scattering has become a standard technique for characterizing such excitations. However, much less was known of the spin-phonon interaction in these compounds from light scattering measurements, which probe the excitations close to the Brillouin zone center (i.e., near zero wave vector). This is because direct interactions of magnons, or higher-lying excitons, with phonons are less likely in the range of wave vectors near the Brillouin zone center accessed in Raman spectroscopy. Nevertheless, such strong interactions have been observed, for example, in $\mathrm{FeCl}_{2} \cdot 2 \mathrm{H}_{2} \mathrm{O}, \mathrm{CsCoCl}_{3}$, and $\mathrm{RbCoCl}_{3}$, where there is an accidental near-degeneracy of the magnon/exciton and phonon frequencies $[1,3,4]$.

More generally, spin-phonon interactions manifest themselves through modifications to the normal temperature dependences of the optic phonons. The exchange cou- pling between magnetic ions influences the phonon frequency, integrated intensity, and linewidth. Such spindependent effects have been reported earlier on in the phonon Raman spectra of, for example, $\mathrm{KCoF}_{3}, \mathrm{VI}_{2}, \mathrm{CsCoBr}_{3}$, EuSe, EuTe, EuO, EuS, $\mathrm{CdCr}_{2} \mathrm{~S}_{4}$, and $\mathrm{CdCr}_{2} \mathrm{Se}_{4}$ [1,5]. The transition metal halides including the perovskite (cubic) structure fluorides, rutile (tetragonal) structure fluorides, and trigonal structure chlorides, bromides, and iodides have produced a rich vein of spin-phonon interactions amenable to detailed study [1]. The observation through Raman scattering of such magnon-phonon interactions via the phonon behavior as a function of temperature has become ubiquitous today.

The basic mechanism assumed for the spin-phonon coupling in the rutile-structure antiferromagnets arises from a spatial modulation of the dominant exchange parameter due to the relative displacements of the atoms corresponding to the phonon modes, as first proposed by Akhiezer [6]. This effect can lead to a modification of both the phonon and magnon dispersion relations, and has been studied theoretically for ferromagnets [7-9] and antiferromagnets $[10,11]$. In this work an extended version of the approach used in our previous spin-phonon calculations [12,13], and applied primarily to $\mathrm{FeF}_{2}$ and $\mathrm{MnF}_{2}$, will be followed. Other forms of spin-phonon coupling, such as those due to magnetoelastic interactions [14,15], are not relevant to the present studies. 
For the phonons in transition metal fluorides with the rutile structure, which are the main concern of this work, experimental results and comparisons with theory have previously been reported for the antiferromagnets $\mathrm{FeF}_{2}$, $\mathrm{MnF}_{2}$, and $\mathrm{NiF}_{2}$ [12,13,16-19], and the diluted antiferromagnet $\mathrm{Fe}_{1-\chi} \mathrm{Zn}_{x} \mathrm{~F}_{2}$ [20]. These compounds possess four zone-center Raman-active optic phonons, all of different symmetries, and because of their simple magnetic structures are ideal candidates for investigating the spin-phonon interaction in antiferromagnets. The Raman-active phonon frequencies, linewidths, and intensities of these materials are affected to varying extents by the antiferromagnetic ordering, and from the extensive experimental data spinphonon coupling coefficients have been deduced for $\mathrm{FeF}_{2}$, $\mathrm{MnF}_{2}$, and $\mathrm{NiF}_{2}[13,19]$. It is of interest to determine the magnitudes of such effects, because they can also influence the magnon Raman scattering [10].

Here, in this overview of the spin-phonon effects in these four relatively simple antiferromagnets, we augment this previous work with additional experimental data for $\mathrm{FeF}_{2}$, $\mathrm{MnF}_{2}$, and $\mathrm{CoF}_{2}$ together with a comprehensive theoretical interpretation and analysis, as pledged in [13]. Significant difference are highlighted (and accounted for) in the cases of $\mathrm{CoF}_{2}$ and $\mathrm{NiF}_{2}$ compared with $\mathrm{FeF}_{2}$ and $\mathrm{MnF}_{2}$. Our study of diverse rutile-structure antiferromagnets provides new insights into the processes and mechanisms involved in spin-phonon effects for different phonons, and different spin values, spin alignments, and spin-orbit coupling strengths.

\section{Experimental results}

\section{1. $\mathrm{FeF}_{2}, \mathrm{MnF}_{2}$, and $\mathrm{NiF}_{2}$}

Detailed experimental investigations of spin-phonon interactions in $\mathrm{FeF}_{2}, \mathrm{MnF}_{2}$, and $\mathrm{NiF}_{2}$ have been reported previously, as mentioned above $[12,13,19]$. Here we summarize the results obtained. There are four Raman-active vibrational modes in these transition-metal difluorides, and the reported phonon frequencies at low temperature along with their symmetry classifications are given in Table 1 . In particular, new results have been obtained for the $B_{2 g}$ phonon mode in $\mathrm{FeF}_{2}$ at low temperatures, and some representative Raman spectra recorded under the same experimental conditions as reported in our earlier work [17] using laser excitation at $514.5 \mathrm{~nm}$ are shown in Fig. 1. The

Table 1. Frequencies (in $\mathrm{cm}^{-1}$ ) and symmetries of the Ramanactive phonon modes at zero wave vector in transition-metal difluorides at low temperature $(\sim 10 \mathrm{~K})$

\begin{tabular}{c|c|c|c|c}
\hline \hline \multirow{2}{*}{$\begin{array}{c}\text { Mode } \\
\text { symmetry }\end{array}$} & \multicolumn{4}{|c}{ Frequency, $\mathrm{cm}^{-1}$} \\
\cline { 2 - 5 } & $\mathrm{FeF}_{2}[13]$ & $\mathrm{MnF}_{2}[13]$ & $\mathrm{NiF}_{2}[19]$ & $\mathrm{CoF}_{2}$ \\
\hline \hline$A_{1 g}$ & 346.8 & 346.9 & 414.5 & 371.2 \\
\hline$B_{1 g}$ & 68.8 & 55.9 & 68.7 & 65.2 \\
\hline$B_{2 g}$ & 505.6 & 480.4 & 541.0 & 517.4 \\
\hline$E_{q}$ & 261.7 & 246.6 & 308.0 & 255.3 \\
\hline \hline
\end{tabular}

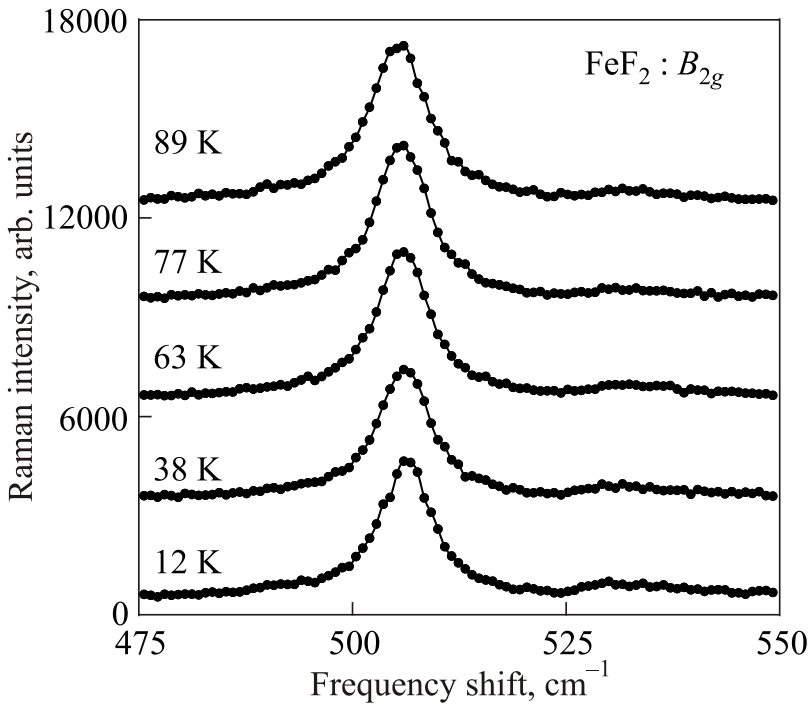

Fig. 1. Raman spectrum of the $B_{2 g}$ phonon mode in $\mathrm{FeF}_{2}$ recorded at several different temperatures below and above its Néel temperature $\left(T_{N}\right)$ of $78 \mathrm{~K}$. The curves are offset vertically for clarity.

$B_{2 g}$ mode frequency, which is much higher than for the other three Raman-active modes in rutile-structure compounds (see Table 1), occurs at $505.6 \mathrm{~cm}^{-1}$ in $\mathrm{FeF}_{2}$ at low temperatures. Figure 1 exhibits another weaker, and asymmetric, Raman peak at $\sim 530 \mathrm{~cm}^{-1}$ that becomes even weaker compared to the $\mathrm{B}_{2 g}$ mode at higher temperatures. This peak is assigned to a phonon combination band associated with the $E_{g}$ mode. At $10 \mathrm{~K}$ the overtone of the $E_{g}$ mode at zero wave vector is expected (using the datum in Table 1 ) to be near $2 \times 261.7=523.4 \mathrm{~cm}^{-1}$, which is above the frequency of the $B_{2 g}$ mode but lower than that of the additional peak at $530 \mathrm{~cm}^{-1}$. This indicates that the latter peak arises from pairs of phonons with equal and opposite, but non-zero, wave vector arising from the $E_{g}$ mode dispersion. Results obtained for the temperature dependences of the Raman-active phonon mode frequencies, linewidths, and integrated intensities, respectively, of these three compounds are illustrated in Figs. 2-4 for $\mathrm{FeF}_{2}$, in Figs. 5 and 6 for $\mathrm{MnF}_{2}$, and in Figs. 7-9 for $\mathrm{NiF}_{2}$.

\section{2. $\mathrm{CoF}_{2}$}

Although general studies of the Raman scattering from phonons in $\mathrm{CoF}_{2}$ have been reported previously [21-23], the new experimental results presented here were needed for a detailed investigation of the spin-phonon interaction.

The purplish-red-colored sample of $\mathrm{CoF}_{2}$ was prepared from a single crystal grown at the Clarendon Laboratory, Oxford University, especially for these spin-phonon studies and our earlier one- and two-magnon studies [23,24]. The cuboid sample of dimensions $3.2 \times 2.0 \times 1.7 \mathrm{~mm}$ was cut to expose (001) [ $Z$ axis direction], (110) [X], and (1 $\overline{1} 0)$ [Y] faces, respectively, and these faces were highly polished with $1 \mu \mathrm{m}$ diamond powder. The Raman spectrum was excited 

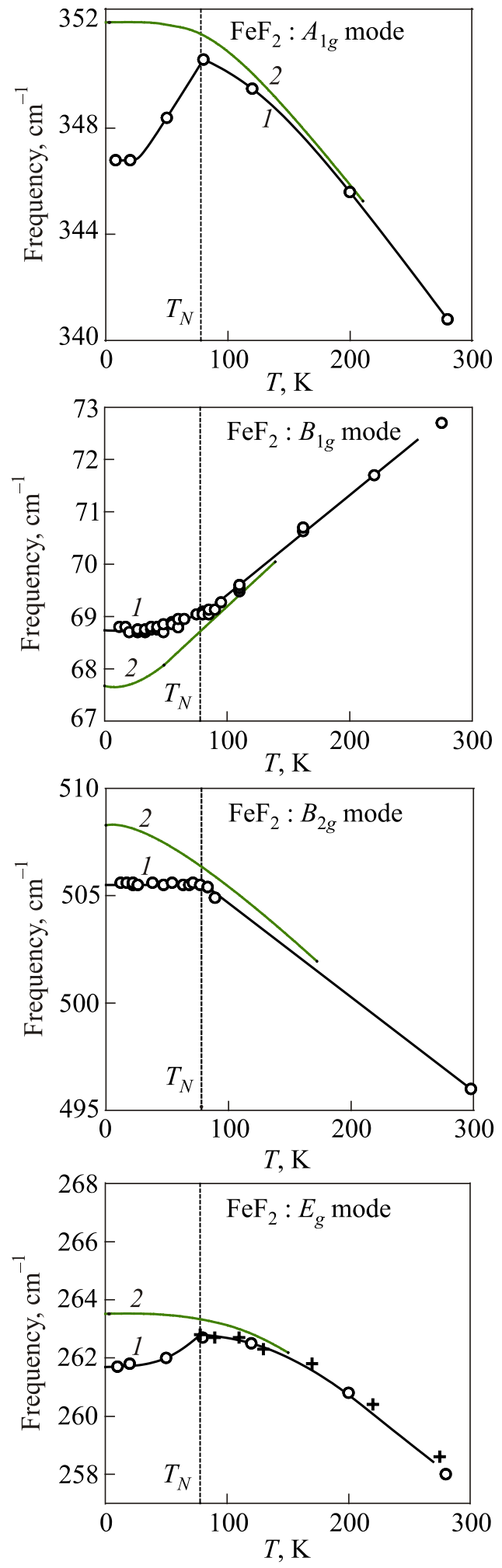

Fig. 2. (Color online) Temperature dependences of the frequencies of the $A_{1 g}, B_{1 g}, B_{2 g}$ and $E_{g}$ Raman-active phonons in $\mathrm{FeF}_{2}$ (following [13]). For the $E_{g}$ mode, the data points marked with a cross are taken from Ref. 16. The black solid lines (1) and the green chain lines (2) are guides to the eye for the experimental data points and for the expected behavior in the absence of spin-phonon coupling (see later theory), respectively.
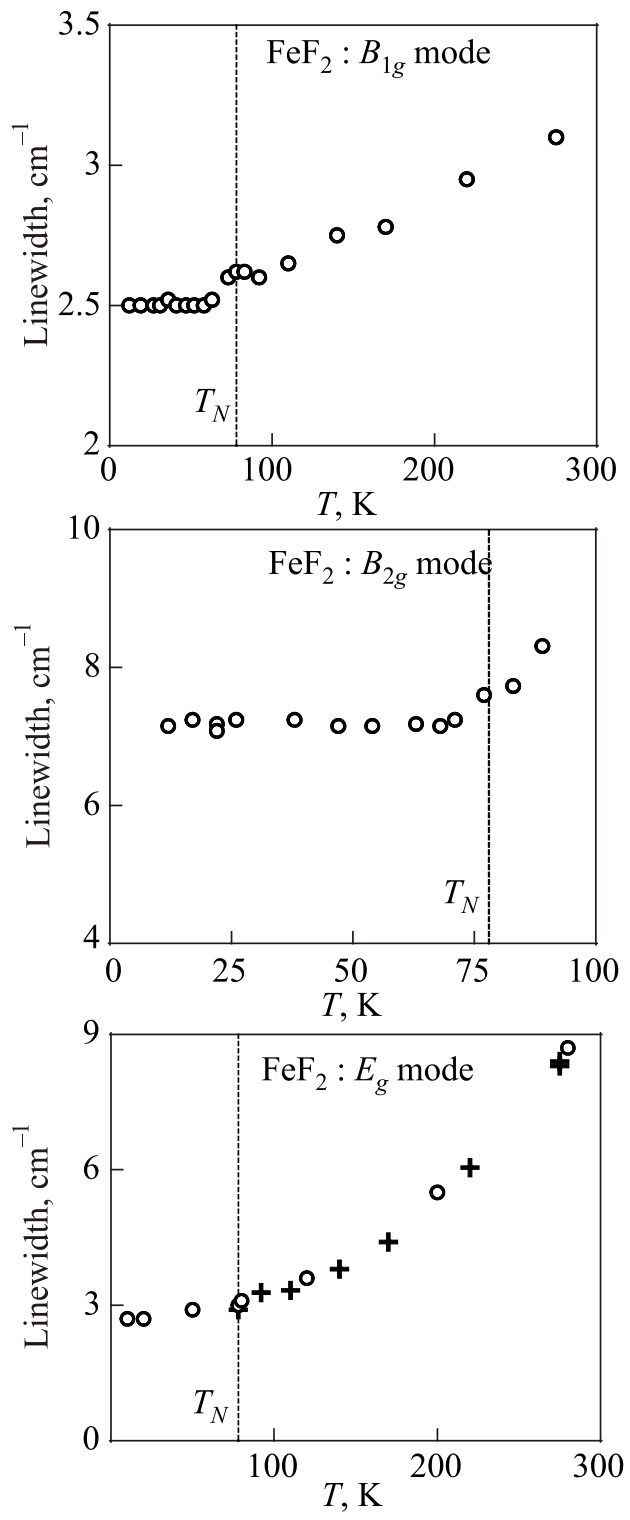

Fig. 3. Temperature dependences of the linewidths (full width at half maximum) of the $B_{1 g}, B_{2 g}$ and $E_{g}$ Raman-active phonons in $\mathrm{FeF}_{2}$. The spectrometer resolution was $2.5 \mathrm{~cm}^{-1}$. For the $E_{g}$ mode, the data points marked with a cross are taken from Ref. 16.

with 500-600 $\mathrm{mW}$ of Ti:sapphire laser light at $800 \mathrm{~nm}$, which avoided any optical absorption [25], analyzed with a Spex 14018 double monochromator at a spectral resolution of $(3.2 \pm 0.1) \mathrm{cm}^{-1}$ unless otherwise indicated, and detected by a cooled RCA 31034A photomultiplier. The sample was mounted in the helium exchange-gas space of a Thor S500 continuous flow cryostat, where the temperature could be controlled to within $0.1 \mathrm{~K}$ and was measured with a gold-iron/chromel thermocouple clamped to the sample. Spectra were recorded in the $90^{\circ}$ scattering geometry. The phonon features were investigated at temperatures up to room temperature. 

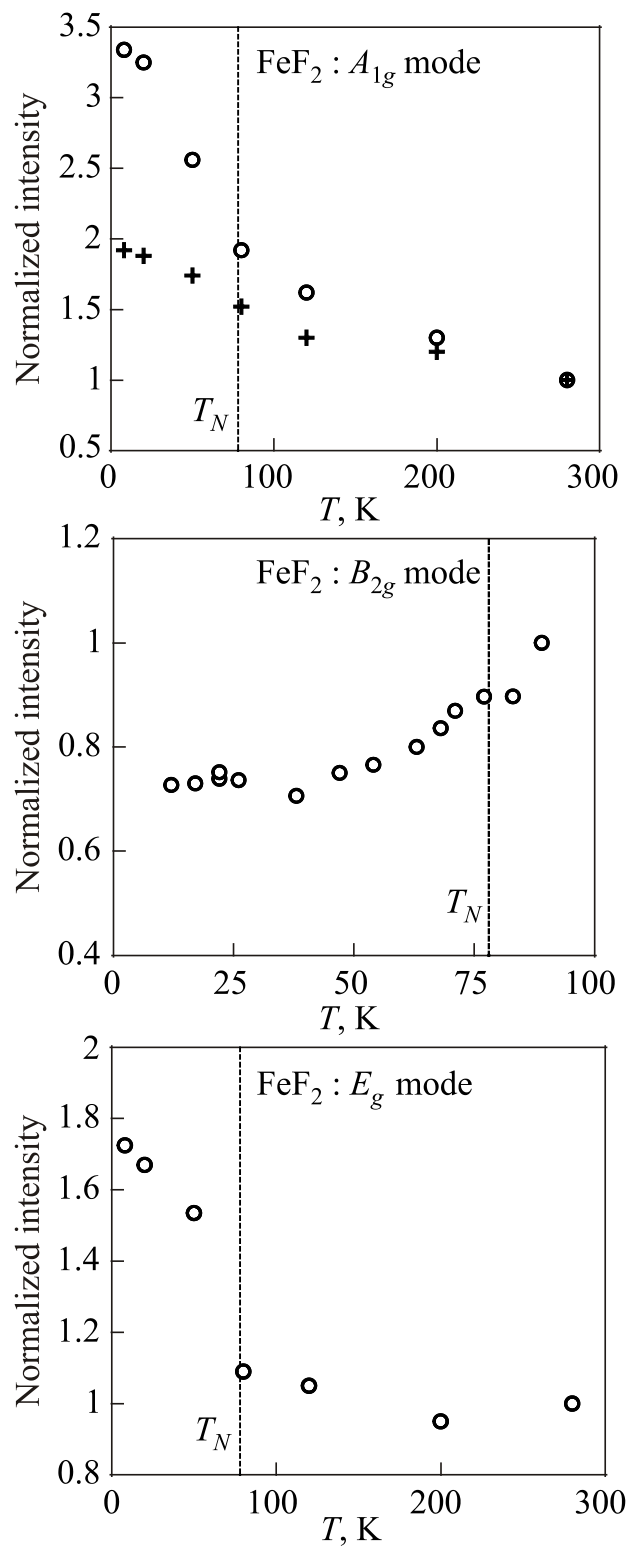

Fig. 4. Temperature dependences of the integrated intensities of the $A_{1 g}, B_{2 g}$ and $E_{g}$ Raman-active phonons in $\mathrm{FeF}_{2}$. The intensities are normalized to the highest temperature in each case. For the $A_{1 g}$ mode, the Raman scattering intensities are shown for (XX) [o] and (ZZ) [+] polarizations.

The polarization dependence of the Raman spectrum of antiferromagnetic $\mathrm{CoF}_{2}$ at low temperature is shown in Fig. 10. The spectra show that depolarization effects due to imperfect experimental conditions are small; see, for example, the extreme weakness of the $A_{1 g}$ mode appearing in the 'forbidden' $X(Z X) Y$-polarized spectrum. Note also that there is no sign of the $B_{2 g}$ mode in the $X(Y X) Y$-polarized spectrum. This is because of the orientation of the crystal $a$ and $b$ axes, which are rotated by $45^{\circ}$ in the $X-Y$ plane away from the $X$ and $Y$ axes. It implies that the $B_{2 g}$ mode is now to be expected in $(X X)$ and $(Y Y)$ polarizations (see Fig. 11). Likewise, the $B_{1 g}$ mode is expected in $(Y X)$ and $(X Y)$ polarizations. These spectra exhibit a sharp peak at $37.0 \mathrm{~cm}^{-1}$
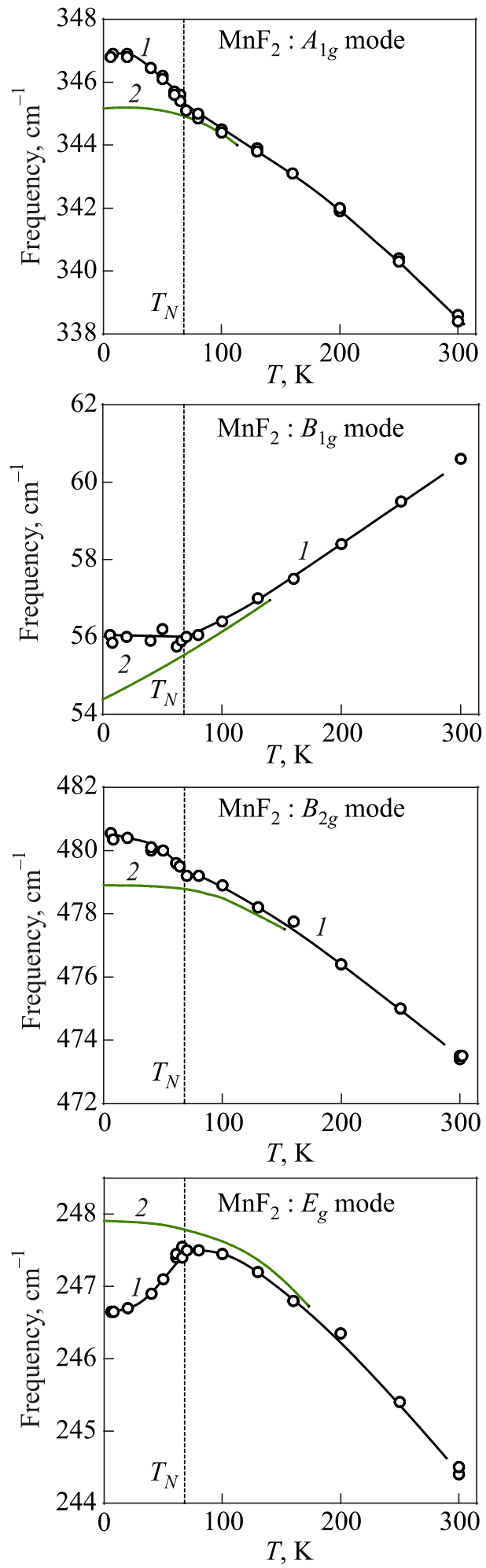

Fig. 5. Temperature dependences of the frequencies of the $A_{1 g}$, $B_{1 g}, B_{2 g}$ and $E_{g}$ Raman-active phonons in $\mathrm{MnF}_{2}$ (following [13]). The lines have the same meaning as in Fig. 2.

that is the lowest lying exciton (conventionally referred to as the magnon) of the ground state multiplet of the $\mathrm{Co}^{2+}$ ion in the exchange field: other excitons can be seen at higher frequencies [23,24]. The one-magnon scattering is observed only in off-diagonal polarizations. The tempera- 

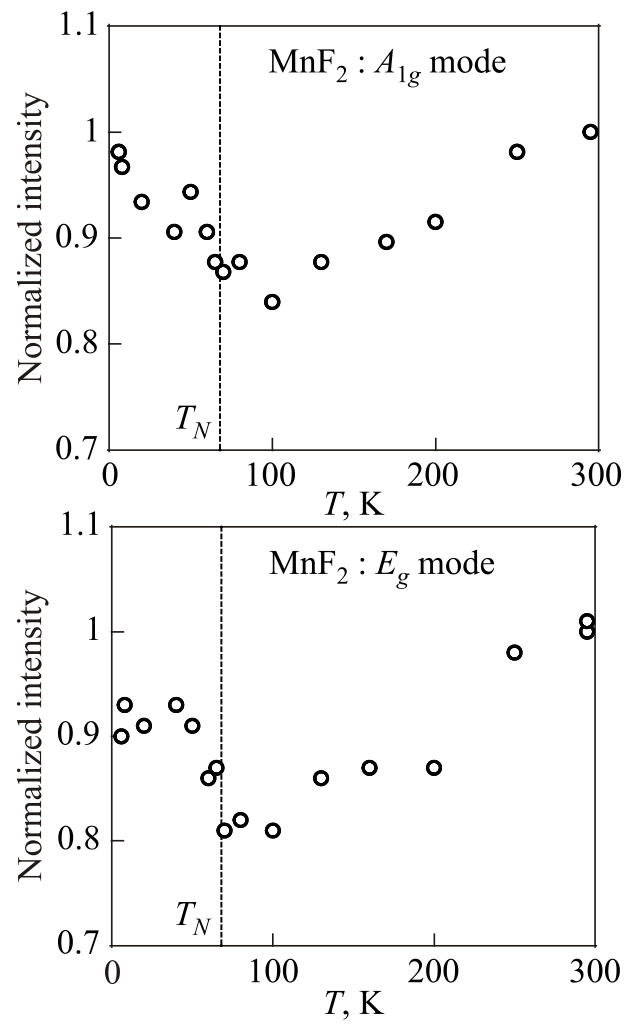

Fig. 6. Temperature dependences of the integrated intensities of the $A_{1 g}$ [in (XX) polarization] and $E_{g}$ Raman-active phonons in $\mathrm{MnF}_{2}$.

ture dependences of the spectra for these same polarizations are next given in Fig. 11, where it can be seen that the phonon peaks vary in frequency and increase in width with increasing temperature in a range extending from well below $T_{N}$ up to and above $T_{N}$, while their peak intensities also vary considerably.

The phonon spectra as a function of temperature and polarization were all readily fitted with a Gaussian/Lorentzian line shape model [26], as shown for example in Fig. 12, and the results obtained for the line parameters in Stokes scattering versus temperature are given in Figs. 13 to 15 . In the case of the $B_{2 g}$ mode spectral region (see Fig. 10), an additional weak Raman band is seen at $\sim 525 \mathrm{~cm}^{-1}$ at low temperature and needs to be accommodated by adding an extra band to the fit, as shown in Fig. 12. As was mentioned earlier for the case for $\mathrm{FeF}_{2}$, this additional band is a second-order combination band arising from the $E_{g}$ mode and it is hardly noticeable for temperatures well above $T_{N}$. In this case, at $10 \mathrm{~K}$ the zone-center overtone of the $E_{g}$ mode is expected (from Table 1) at $2 \times 255.3=510.6 \mathrm{~cm}^{-1}$, which implies that the band at $525 \mathrm{~cm}^{-1}$ must arise from pairs of non-zero wave vector phonons like in $\mathrm{FeF}_{2}$.

It is noticeable from Figs. 13-15 that the results obtained for $\mathrm{CoF}_{2}$ differ in several aspects from those of the other three rutile-structure antiferromagnets, presumably because of the strong effect of the spin-orbit interaction in $\mathrm{CoF}_{2}$ and through the presence of other electronic excitations (higherlying excitons) [22], as will be discussed later. However, the
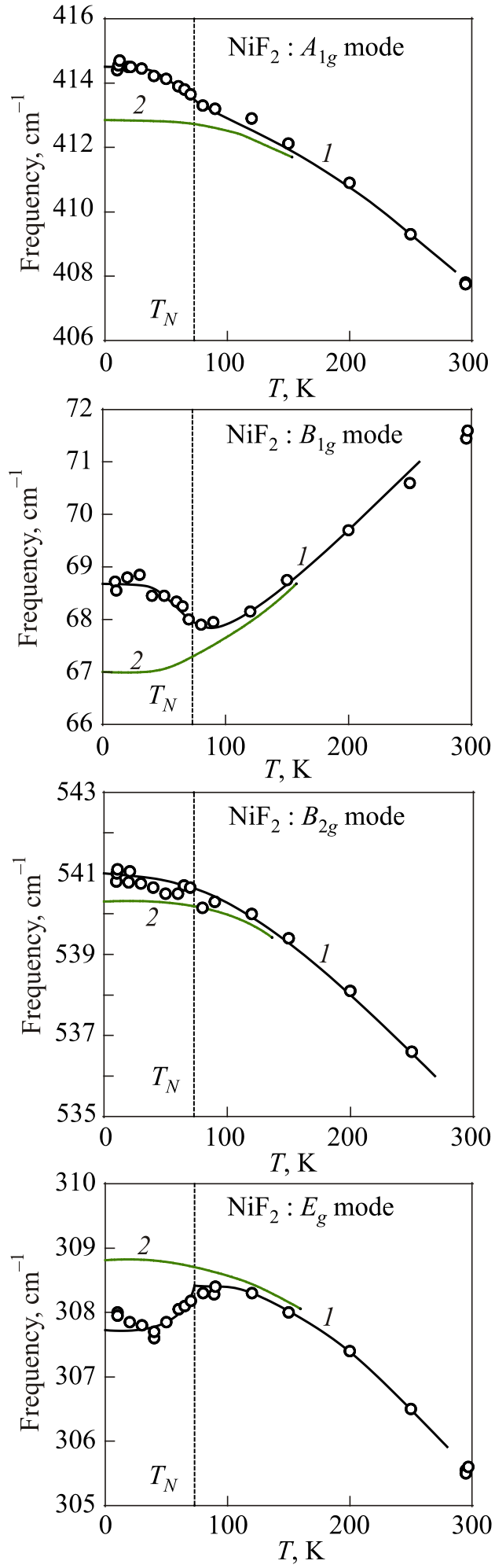

Fig. 7. Temperature dependences of the frequencies of the $A_{1 g}$, $B_{1 g}, B_{2 g}$, and $E_{g}$ Raman-active phonons in $\mathrm{NiF}_{2}$ (following [19]). The lines have the same meaning as in Fig. 2.

$B_{1 g}$ mode softening with decreasing temperature from room temperature down to the Néel temperature $T_{N}$, as shown in Fig. 13, is analogous to that found earlier in the other transition metal fluorides (see, for example, Figs. 2 and 7) and is ascribed to the anisotropic thermal contraction of the crystal lattice on cooling [19,27]. 

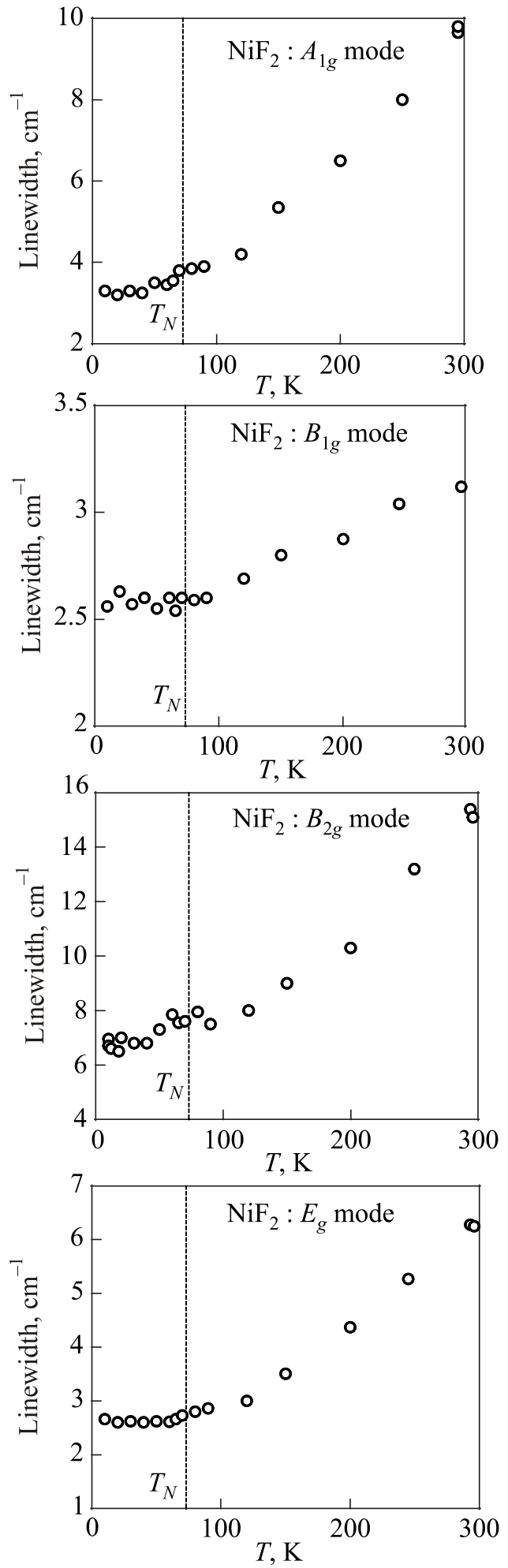

Fig. 8. Temperature dependences of the linewidths of the $A_{1 g}$, $B_{1 g}, B_{2 g}$, and $E_{g}$ Raman-active phonons in $\mathrm{NiF}_{2}$ (following [19]). The spectrometer resolution was $2.5 \mathrm{~cm}^{-1}$.

The phonon linewidths for the $A_{1 g}, B_{1 g}$, and $B_{2 g}$ modes (see Fig. 14) show little variation with temperature below $T_{N}$, similar to what was found for the these modes in $\mathrm{FeF}_{2}$ and $\mathrm{MnF}_{2}$. It should be noted, however, that the $B_{1 g}$ mode linewidth is resolution limited at low temperatures. In that regard, high-resolution Raman scattering
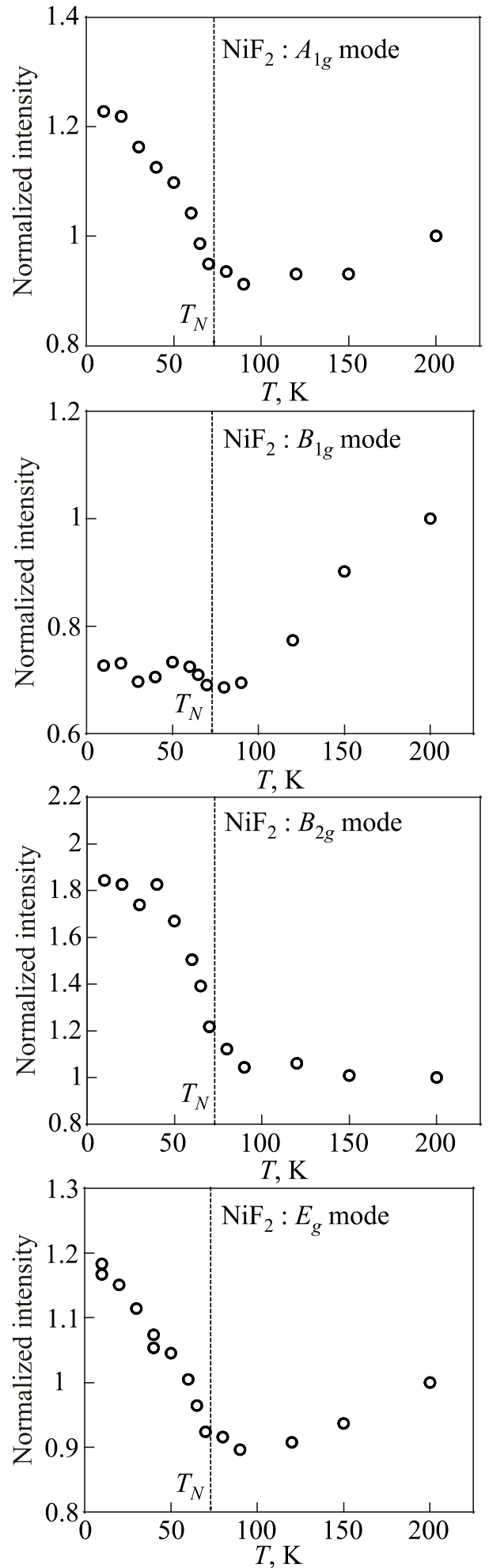

Fig. 9. Temperature dependences of the integrated intensities of the $A_{1 g}$ [in $(X X)$ polarization], $B_{1 g}, B_{2 g}$, and $E_{g}$ Raman-active phonons in $\mathrm{NiF}_{2}$ (following [19]).

measurements have revealed that the true linewidth of the $E_{g}$ mode in $\mathrm{FeF}_{2}\left(\mathrm{NiF}_{2}\right)$ at $0 \mathrm{~K}$ is $0.6(0.5) \mathrm{cm}^{-1}$ [28]. The $E_{g}$ mode exhibits a striking increase in linewidth below $T_{N}$ (note also that this line becomes very sharp at $T_{N}$ ) that has not been observed for the other fluorides. This anomalous behavior is attributed to a resonant increase in an- 

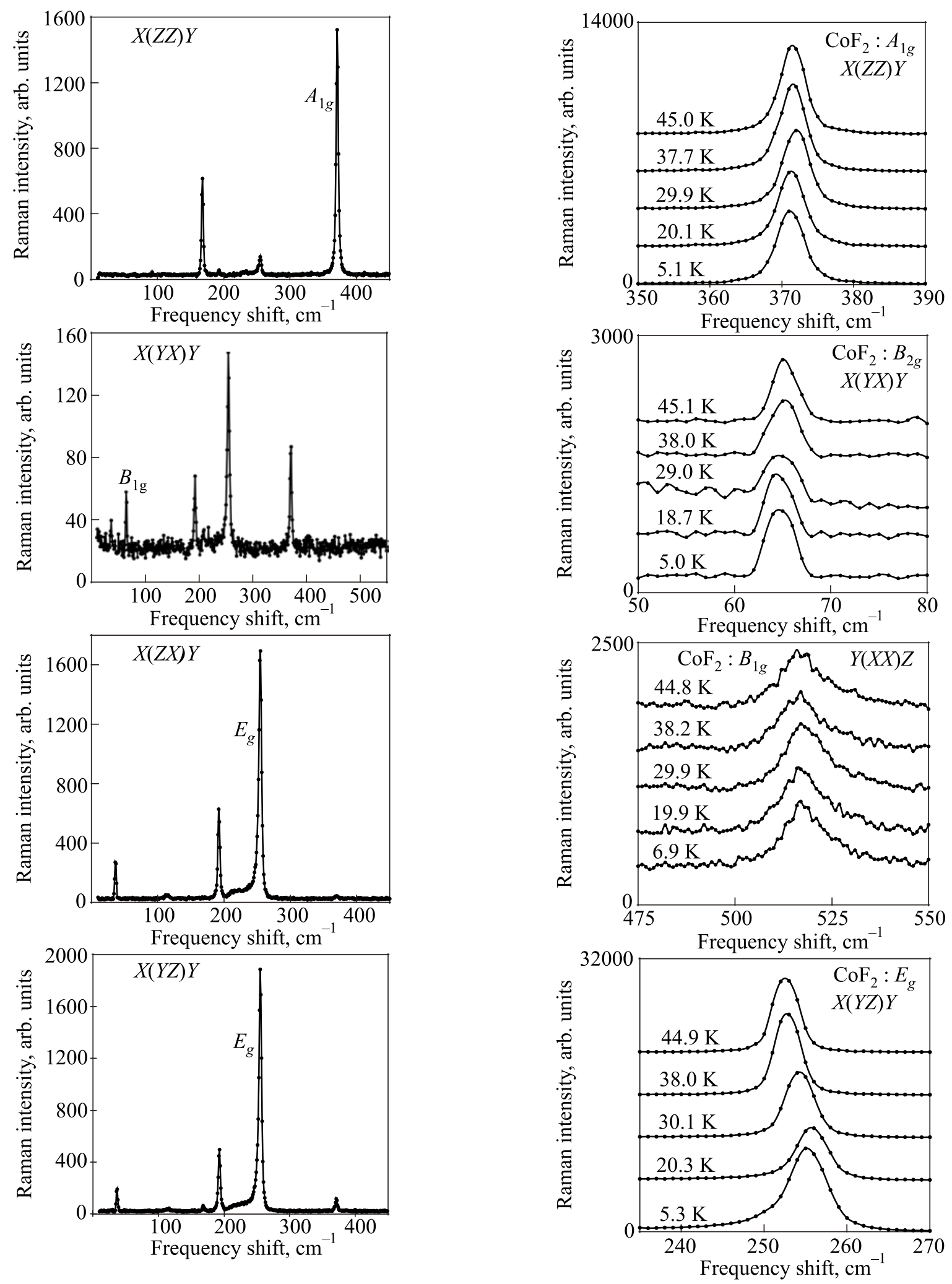

Fig. 10. Polarization dependences of the Raman spectrum of antiferromagnetic $\mathrm{CoF}_{2}$ at $10 \mathrm{~K}$. The spectral resolution was $2.4 \mathrm{~cm}^{-1}$. The first-order Raman-active phonons are identified by their mode symmetries.

harmonic coupling with other lower-frequency phonons and/or excitons consequent upon the continued sharp increase in the phonon frequency with decreasing temperature (see Fig. 13).

Fig. 11. The temperature dependences at low temperatures of the four phonon modes in the $\mathrm{CoF}_{2}$ Raman spectrum recorded in the polarizations shown in the figures.

The integrated intensities of the various modes (see Fig. 15) either decrease with decreasing temperature below $T_{N}$ (for the $A_{1 g}$ and $E_{g}$ modes) or slightly increase (for the $B_{1 g}$ and $B_{2 g}$ modes). 

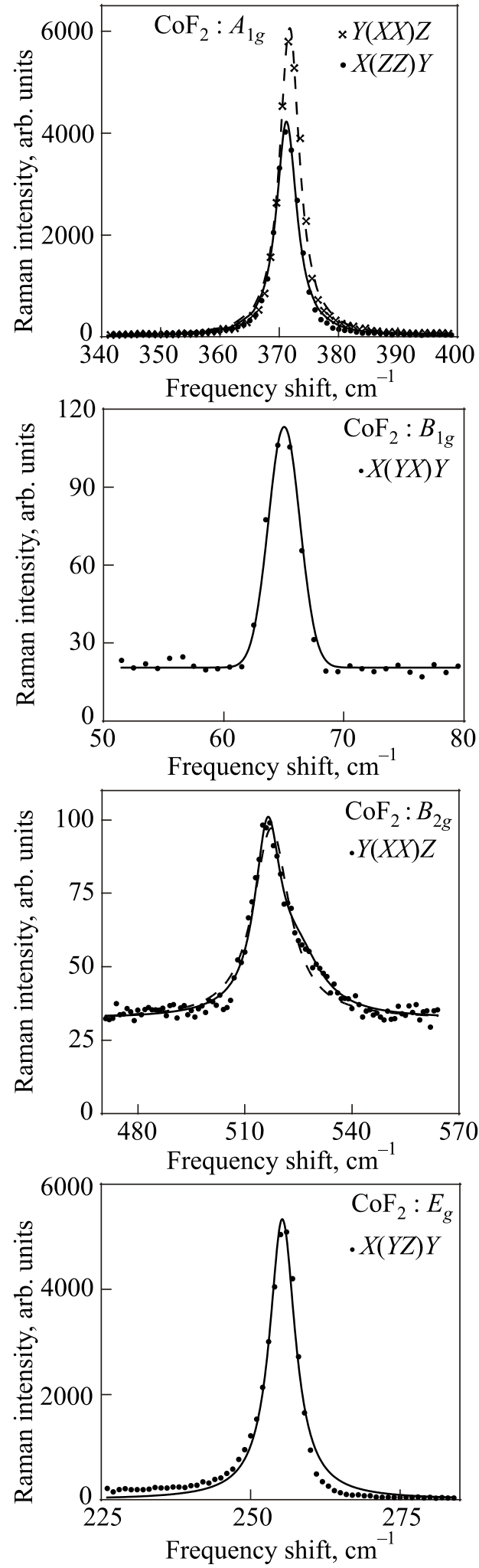

Fig. 12. Fits to the $\mathrm{CoF}_{2}$ phonon spectra recorded at $\sim 10 \mathrm{~K}$ for various polarizations. See text for details.

\section{Theoretical methods}

Here we start with a theoretical approach similar to that used in our earlier spin-phonon studies of the rutile antiferromagnets [12,13,19], which all have the structure shown in Fig. 16. For $\mathrm{FeF}_{2}, \mathrm{MnF}_{2}$ and $\mathrm{CoF}_{2}$ the spins are
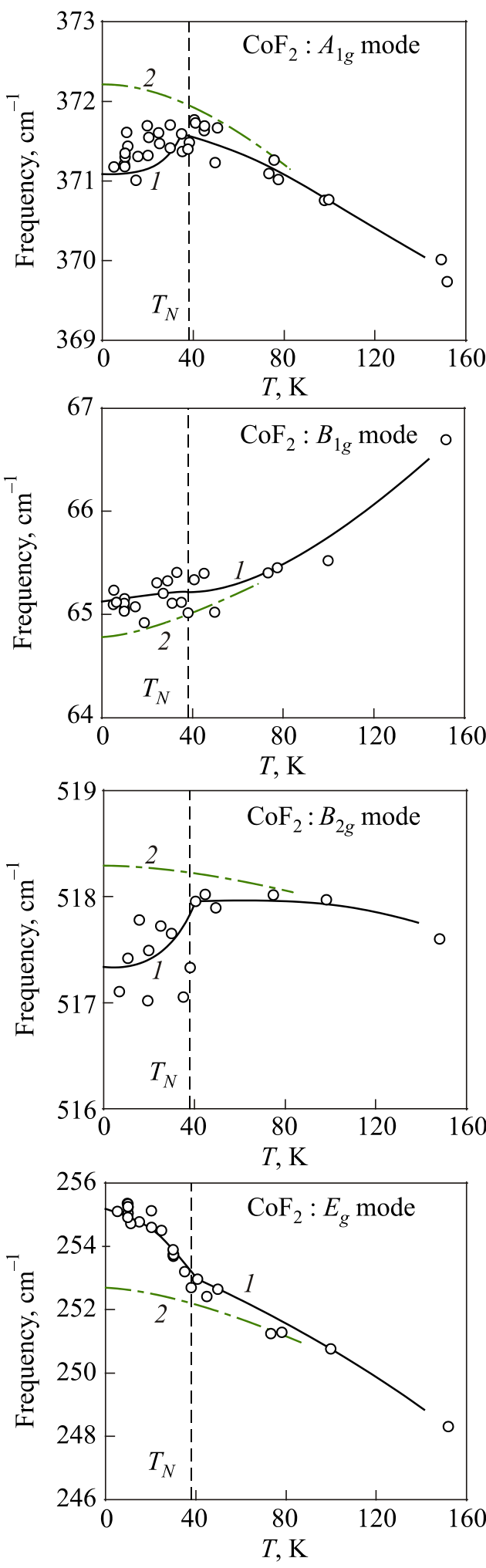

Fig. 13. Temperature dependences of the frequencies of the $A_{1 g}$, $B_{1 g}, B_{2 g}$, and $E_{g}$ Raman-active phonons in $\mathrm{CoF}_{2}$. The lines have the same meaning as in Fig. 2.

aligned parallel and antiparallel to the crystal $c$ axis (as shown), but in $\mathrm{NiF}_{2}$ the spin alignment is in the $a b$ plane with a very slight canting away from the principal axes. In all cases we may write the dominant inter-sublattice exchange part of the magnetic Hamiltonian as 

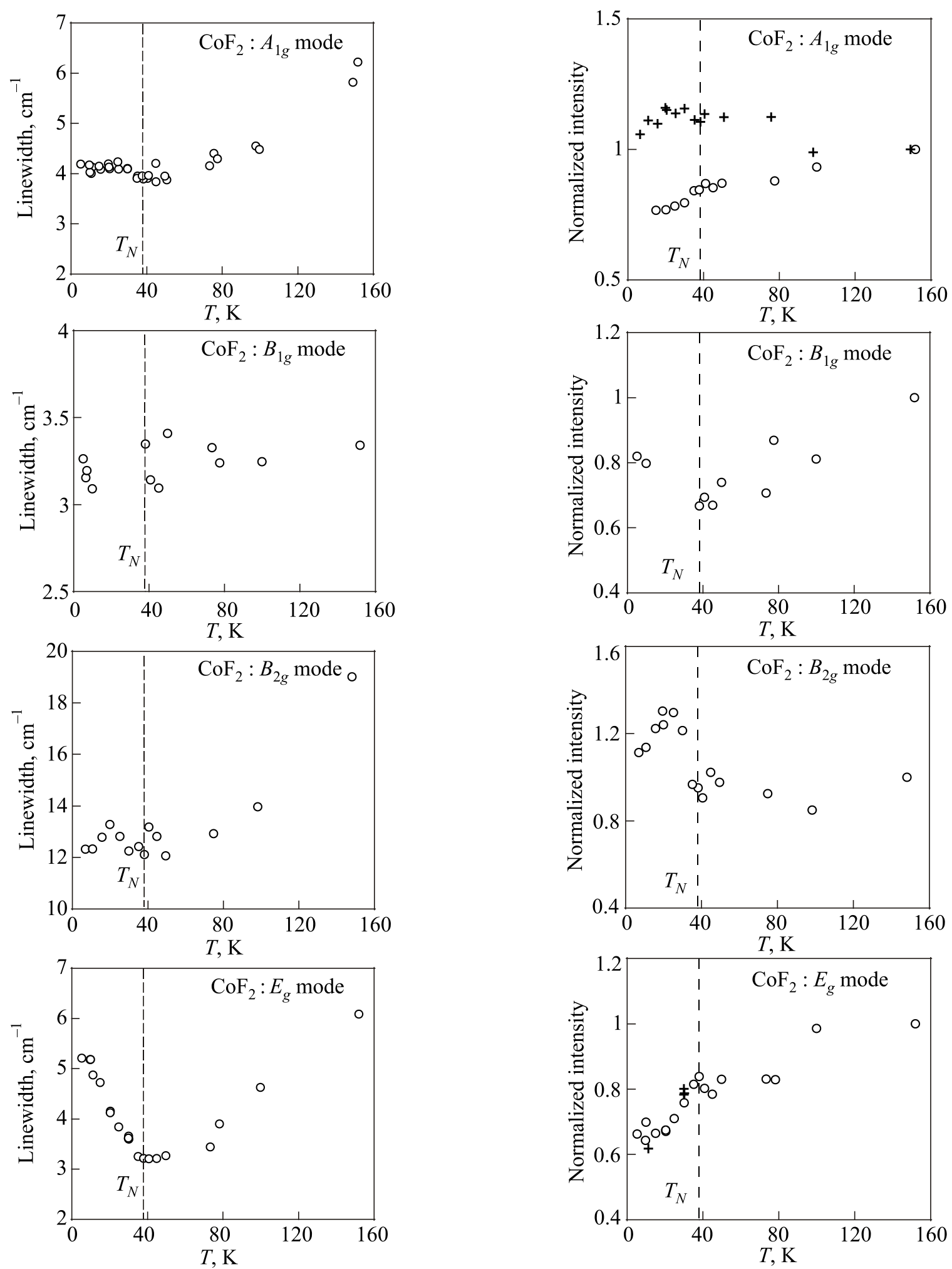

Fig. 14. Temperature dependences of the linewidths of the $A_{1 g}$, $B_{1 g}, B_{2 g}$, and $E_{g}$ Raman-active phonons in $\mathrm{CoF}_{2}$. The spectrometer resolution was $3.2 \mathrm{~cm}^{-1}$.

$$
H_{\mathrm{ex}}=\sum_{i, j} J_{i j}\left(\mathbf{r}_{1}, \mathbf{r}_{2}, \mathbf{r}_{3}, \mathbf{r}_{4}\right) \mathbf{S}_{i} \cdot \mathbf{S}_{j},
$$

where $i$ and $j$ denote the neighboring magnetic sites on opposite sublattices. As well as the dominant exchange 


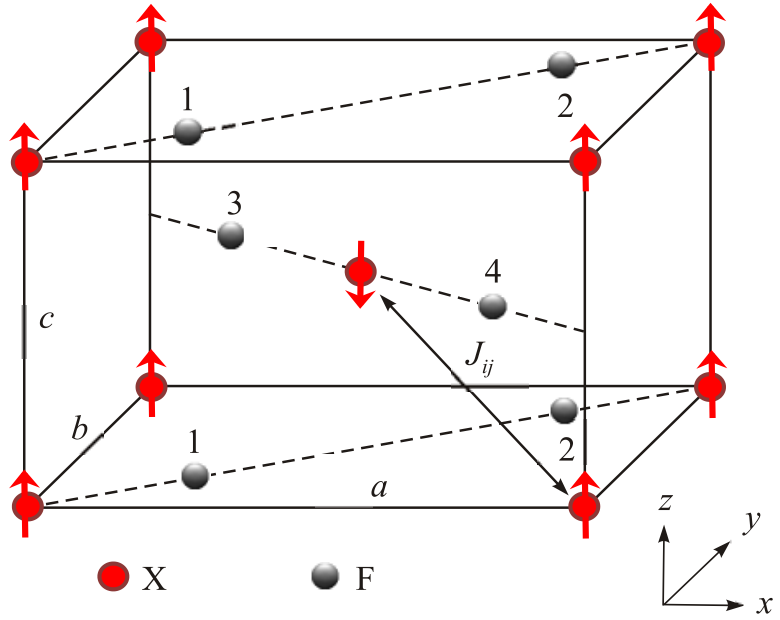

Fig. 16. (Color online) Schematic of the tetragonal rutile unit cell of $\mathrm{XF}_{2}(\mathrm{X}=\mathrm{Fe}, \mathrm{Mn}, \mathrm{Ni}$, or $\mathrm{Co})$, showing the dominant intersublattice exchange $J_{i j}$ and the labeling of four $\mathrm{F}$ atoms relative to the body-centered magnetic site. We have $a=b \neq c$. In the case of $\mathrm{NiF}_{2}$ the spin alignments are different (see text).

indirect process of superexchange $[1,29]$. In rutilestructure materials the $\mathrm{F}$ atoms become displaced due to the phonon modes, hence modulating the exchange term. In fact, the four Raman-active modes leave the magnetic atoms stationary, while producing displacements in $\mathbf{r}_{n}$ that can be written down following [29]. Then a Taylorseries expansion in terms of these displacements yields a leading-order spin-phonon coupling Hamiltonian that is quadratic in the spin operators and linear in the phonon operators (see, e.g., [6-11]). The effects of the weaker intra-sublattice exchange [1] on the phonon modes in the rutile antiferromagnets will be neglected here, but it is important to include the magnetic single-ion anisotropy terms in the full Hamiltonian, since these are relatively large for some of the materials studied here.

\subsection{Phonon frequency shifts}

In lowest order of perturbation theory the result for the renormalized phonon frequency $\omega_{\mathrm{ph}}$ for any mode can be expressed as [12,13]

$$
\omega_{\mathrm{ph}}=\omega_{\mathrm{ph}}^{0}+\lambda\left\langle\mathbf{S}_{i} \cdot \mathbf{S}_{j}\right\rangle .
$$

Here $\omega_{\mathrm{ph}}^{0}$ denotes the phonon frequency in the absence of the spin-phonon coupling, and $\left\langle\mathbf{S}_{i} \cdot \mathbf{S}_{j}\right\rangle$ is a statistical average involving the neighboring spins on opposite sublattices. The factor $\lambda$, which may be positive or negative, takes a different value for each phonon mode and is of second order in the spin-phonon interaction parameters (spatial derivatives of the exchange terms) [10,11]. It is convenient to write the frequency shift as

$$
\Delta \omega_{\mathrm{ph}}(T) \equiv\left(\omega_{\mathrm{ph}}^{0}-\omega_{\mathrm{ph}}\right)=-\lambda S^{2} \Phi(T),
$$

where we define an antiferromagnetic short-range order parameter by

$$
\Phi(T)=-\frac{1}{S^{2}}\left\langle\mathbf{S}_{i} \cdot \mathbf{S}_{j}\right\rangle>0
$$

We next discuss a procedure to evaluate approximately the above quantity, so that a comparison can be made between theory and the experimental data. Results are required for $\Phi(T)$ at temperatures both below and above $T_{N}$, as well as for different values of the spin $S$. First we note that in a mean-field approximation $\Phi(T)$ varies from unity at $T=0$ and decreases monotonically with increasing temperature as $\left(\left\langle S^{z}\right\rangle / S\right)^{2}$, where $\left\langle S^{z}\right\rangle$ is the longitudinal spin average (proportional to the sublattice magnetization) calculated using the appropriate Brillouin function. This result is applicable also for $\mathrm{NiF}_{2}$ provided the canting effects through an angle of order $0.4^{\circ}$ at low temperatures (see, e.g., [1]) are ignored and we now take the superscript $z$ to refer to the new direction of spin alignment.

The above approximation is good for $T<T_{N}$ only, because it ignores the short-range order in the antiferromagnet that becomes important in the vicinity of $T_{N}$ and above. For this latter temperature region we make use of a two-spin cluster method (see, e.g., Ref. 30 for a general account), following in particular the modified form of the constant coupling approximation for antiferromagnets due to Elliott [31]. In the present application this allows us to deduce that

$$
\left\langle\mathbf{S}_{i} \cdot \mathbf{S}_{j}\right\rangle=-S(S+1) /\left(z_{0}-1\right)
$$

as $T$ tends to $T_{N}$ from above. Here $z_{0}$ denotes the number of nearest neighbors on the opposite sublattice for any magnetic site. Hence for rutile-structure antiferromagnets where $z_{0}=8$, we have the remarkably simple approximate result that

$$
\Phi\left(T_{N}\right)=(S+1) / 7 S .
$$

Thus it is predicted that the ratio $\Phi\left(T_{N}\right) / \Phi(0)$ decreases with increasing spin quantum number, ranging in the present applications from approximately 0.43 for $\mathrm{CoF}_{2}$ ( $S=1 / 2)$, to 0.29 for $\mathrm{NiF}_{2}(S=1)$, to 0.21 for $\mathrm{FeF}_{2}(S=2)$, and to 0.20 for $\mathrm{MnF}_{2}(S=5 / 2)$. The latter two ratios (for the larger $S$ values) agree accurately with the values computed in $[12,13]$ without the use of Eq. (6), and thus validate this new method of calculating $\Phi(T)$ at $T_{N}$ and above. Using the same approach, it is found that the temperature dependence for the magnetic susceptibility above $T_{N}$ is given by

$$
\Phi(T) \propto\left(\frac{2 T_{N}}{T_{N}+T}\right)^{2}
$$

Therefore we conclude that $\Phi(T)$ decreases monotonically with temperature, falling at $T=2 T_{N}$ (for example) to a fraction $4 / 9$ of its value at $T_{N}$. 


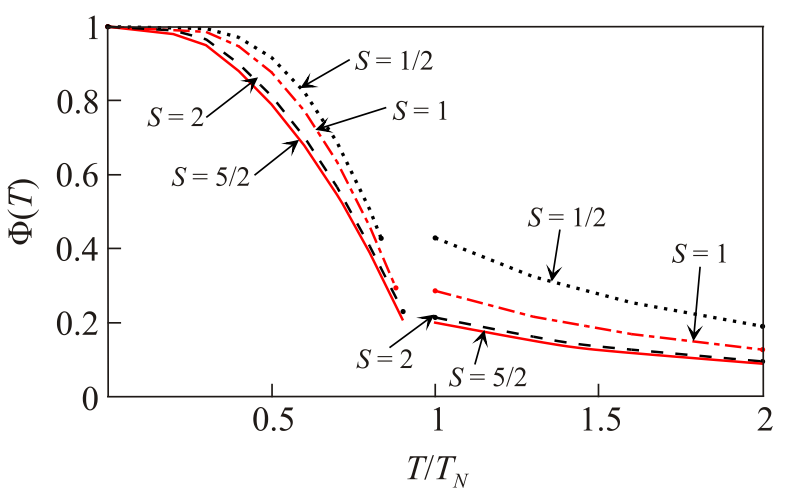

Fig. 17. Numerical estimates for the normalized short-range order parameter $\Phi(T)$ as a function of reduced temperature. Curves are shown for rutile-structure antiferromagnets with spin $S=1 / 2,1,2$, and $5 / 2$.

The general results obtained on calculating $\Phi(T)$ from zero temperature up to twice $T_{N}$ are shown in Fig. 17. We employed mean-field theory for the estimates at $T<T_{N}$, as for $[12,13]$, and the new two-spin cluster approach above $T_{N}$. It is evident, however, that the new results for $S$ equal to $1 / 2$ and 1 are quite distinct from those for the higher spin values, where there is a larger high-temperature "tail". The consequences of this for interpretation of the spinphonon experimental data will be discussed in Sec. 4.

\subsection{Phonon Raman intensities}

The analytical and numerical results for $\Phi(T)$ are also useful for the interpretation of the Raman scattering integrated intensities. For this application the conventional approach (see, e.g., [1,32]) is to expand the polarizability tensor in terms of both the spin operators for the magnetic sites and the displacement operators for the $\mathrm{F}$ atoms. By analogy with studies for ferromagnetic materials [16,33], the spin-dependent Stokes intensity due to the phonons in the present case can be expressed as [12,19]

$$
I(T)=\left(n_{\mathrm{ph}}+1\right)\left[\left|A+B\left\langle\mathbf{S}_{i} \cdot \mathbf{S}_{j}\right\rangle\right|^{2}+C^{2}\left\langle S^{z}\right\rangle^{2}\right] .
$$

Here $n_{\mathrm{ph}}$ is the Bose thermal population factor for phonons, while $A, B$, and $C$ are magneto-optical constants. Coefficient $A$ provides the sole contribution to the phonon scattering intensity in the absence of spin-phonon coupling and the extra terms involving $B$ and $C$ involve the spin averages.

At temperatures below $T_{N}$ in the mean-field approximation the above result can be rewritten as

$$
I(T)=\left(n_{\mathrm{ph}}+1\right)\left[\left|A-B S^{2} \Phi(T)\right|^{2}+C^{2} S^{2} \Phi(T)\right], \quad T<T_{N},
$$

and so the numerical values obtained for $\Phi(T)$ as in Fig. 17 may be employed. On the other hand, at temperatures above $T_{N}$ we have $\left\langle S^{z}\right\rangle=0$, so the term proportion to $C^{2}$ in Eq. (8) vanishes.

\subsection{Phonon linewidths}

Finally we remark that the phonon linewidths due to damping of the modes are more complicated to interpret because there are various mechanisms that may occur. In general, phonon damping in crystalline solids may arise from three-phonon and four-phonon processes due to anharmonic interactions between atoms. For example, in the three-phonon case a zone-center phonon may split into two other phonons with equal and opposite wave vectors, giving rise to a linewidth. Phonon damping may occur also as a consequence of scattering from any static impurities or lattice defects in the crystal.

Calculations of the phonon damping contributions in antiferromagnets that were specifically due to the spinphonon interactions were made by Cottam [11]. Processes whereby a phonon undergoes either splitting (into two spin waves) or confluence (by absorbing a spin wave and scattering into another spin-wave state) were studied, subject to conservation of overall energy and wave vector. Estimates of the importance of these damping mechanisms were made for a cubic material, but the results were mainly for acoustic phonons. Other phonon damping calculations in magnetic materials were reported by Wakamura [34] as part of an experimental investigation using infrared reflectivity of the ferrimagnet $\mathrm{FeCr}_{2} \mathrm{~S}_{4}$. A large anomaly in the phonon damping was observed close to the Curie temperature of the material and was attributed to spin-phonon effects near the Brillouin-zone boundary for the phonon. This latter mechanism, however, is not applicable to our case.

\section{Comparison of experiment and theory}

\section{1. $\mathrm{FeF}_{2}$ and $\mathrm{MnF}_{2}$}

The compounds $\mathrm{FeF}_{2}$ and $\mathrm{MnF}_{2}$ with spins of $S=2$ and $5 / 2$, respectively, are expected on the basis of numerical results shown in Fig. 17 to exhibit similar variations in their phonon band parameters with temperature. The experimental results given in Figs. 2-6 show that this is indeed generally the case.

As noted earlier, the new theoretical results obtained here for the behavior of $\Phi(T)$ for temperatures $T>T_{N}$ are identical to those found earlier following a different approach [12,13]. We have carried out a re-analysis of the experimental data for the phonon frequencies, paying particular attention to the high-temperature "tail" (leading to only slight differences for these two compounds), and the estimated values for the spin-phonon interaction parameter are given in Table 2. Here it has been convenient (in view of comparisons to be made with the other compounds) for the results to be scaled as $\lambda S^{2}$, as in Eq. (3). 
Table 2. Scaled spin-phonon coupling coefficients, $\lambda S^{2}$ (in $\mathrm{cm}^{-1}$ ), for each Raman-active phonon mode in the rutile-structure antiferromagnets as deduced from the phonon frequency versus temperature behavior

\begin{tabular}{c|c|c|c|c|c}
\hline \hline Compound & Spin $S$ & $\begin{array}{c}\lambda S^{2} \\
\text { for } A_{1 q}\end{array}$ & $\begin{array}{c}\lambda S^{2} \\
\text { for } B_{1 q}\end{array}$ & $\begin{array}{c}\lambda S^{2} \\
\text { for } B_{2 q}\end{array}$ & $\begin{array}{c}\lambda S^{2} \\
\text { for } E_{q}\end{array}$ \\
\hline \hline $\mathrm{MnF}_{2}$ & $5 / 2$ & -1.9 & -1.9 & -1.9 & 1.2 \\
$\mathrm{FeF}_{2}$ & 2 & -1.2 & -0.3 & 2.0 & 2.0 \\
$\mathrm{NiF}_{2}$ & 1 & -1.8 & -1.8 & -0.7 & 1.0 \\
$\mathrm{CoF}_{2}$ & $1 / 2$ & 1.0 & -0.3 & 0.9 & 2.0 \\
\hline \hline
\end{tabular}

As can be seen in Fig. 3, the phonon linewidths in $\mathrm{FeF}_{2}$ are scarcely, if at all, affected by the antiferromagnetic ordering for the $B_{1 g}, B_{2 g}$, and $E_{g}$ modes. The linewidth is essentially constant at temperatures below $T_{N}$, and the same is true for the $A_{1 g}$ mode $[12,13,16]$. The results for $\mathrm{MnF}_{2}$ also show no obvious effect of magnetic ordering on the phonon linewidths $[12,13]$. A note of caution needs to be added here for the $B_{1 g}$ mode, which is so sharp that the data are resolution limited at low temperatures (see Fig. 3) and thus some small spinphonon interaction effect might be observable at a much higher instrumental resolution than the one used here.

The integrated intensities of the phonon modes, however, do display variations at low temperatures consistent with a spin-phonon coupling effect, as shown in Figs. 4 and 6. These figures show that the intensities of the $A_{1 g}$ and $E_{\mathrm{g}}$ modes of both $\mathrm{FeF}_{2}$ and $\mathrm{MnF}_{2}$ are considerably enhanced by spin-phonon coupling.

\section{2. $\mathrm{CoF}_{2}$}

The magnetic behavior of cobalt fluoride is quite distinct from $\mathrm{FeF}_{2}$ and $\mathrm{MnF}_{2}$, mainly because of its strong spin-orbit interaction and the existence of a ground state multiplet of $\mathrm{Co}^{2+}$ ion electronic states (or excitons for $T<T_{N}$ ). The latter property usually leads to a simplified theoretical model for $\mathrm{CoF}_{2}$ in terms of it being a Heisenberg antiferromagnet with effective spin $S=1 / 2$ (see, e.g., [1,23,24]. Corresponding to this lowest nonzero value for $S$ we have the case where the largest spin-phonon "tail" persists at $T>T_{N}$ (as seen by the behavior predicted for $\Phi(T)$ in Fig. 17). This property makes the spin-phonon effects less obvious to detect in $\mathrm{CoF}_{2}$, which is what we find experimentally. Also, the estimates for the coupling coefficients $\lambda$ given in Table 2 are likely to be less precise for $\mathrm{CoF}_{2}$.

\section{3. $\mathrm{NiF}_{2}$}

Nickel fluoride with $S=1$ is also different from $\mathrm{FeF}_{2}$ and $\mathrm{MnF}_{2}$, but in this case it is because of its different spin alignment as well as the spin canting that occurs for $T<T_{N}$. Instead of lying along the crystal $c$ axis, the spins in $\mathrm{NiF}_{2}$ lie in the $a b$ plane and are tilted slightly away from the principal axes. This spin canting modifies the magnetic properties of $\mathrm{NiF}_{2}$, giving rise to a lower "ferromagnetic" spin wave branch as well as the usual antiferromagnetic branch found in isomorphous $\mathrm{FeF}_{2}$ and $\mathrm{MnF}_{2}$ [35]. The spin canting is small ( $\sim .4^{\circ}$ away from the $a$ axis), and can be ignored in our spin-phonon analysis since it has negligible effect on the calculation of the pairwise spin average $\Phi(T)$. However, in our earlier work [19] we used approximate numerical values for $\Phi(T)$ as a function of temperature, similar to those used for $\mathrm{FeF}_{2}$ and $\mathrm{MnF}_{2}$. In the present case, given the novel definitive result for $\Phi(T)$ presented here for other values of $S$, new results have been obtained for the spin-phonon coupling constants, based on the experimental frequency dependences on temperature shown in Fig. 7. These values are given for the four phonons in Table 2.

Generally, we find that the phonon linewidth increases steadily with increasing temperature above $\sim 50 \mathrm{~K}$ (see Fig. 8), as is observed in other rutile compounds. However, for the $A_{1 g}, B_{2 g}$, and $E_{g}$ modes a sharp rise in the linewidth of about $20 \%$ of the $10 \mathrm{~K}$ value is evident at temperatures near $T_{N}$. These linewidth anomalies have not been seen in $\mathrm{MnF}_{2}$ and $\mathrm{FeF}_{2}$. The $B_{1 g}$ line is very sharp at all temperatures, and no anomaly was detected in the linewidth at temperatures near $T_{N}$ within the experimental uncertainty. Usually, when considering anharmonic phonon decay, the higher the phonon frequency the broader the Raman line, as observed here for $\mathrm{NiF}_{2}$ (see Fig. 8), owing to the higher number of available decay paths into phonons with equal and opposite wave vector. The low frequency $B_{1 g}$ mode can only decay into acoustic phonon modes and hence its extreme sharpness even at room temperature. For the phonons in antiferromagnets below $T_{N}$, an additional decay channel is possible into pairs of magnons of equal and opposite wave vector $[9,11,12]$. However, in $\mathrm{NiF}_{2}$ a lowfrequency "ferromagnetic" magnetic branch exists, as mentioned earlier. It is thus possible that phonon decays into this branch are responsible for the observed changes in linewidth of the $A_{1 g}, B_{2 g}$, and $E_{g}$ modes in $\mathrm{NiF}_{2}$ near $T_{N}$. On the other hand, the $B_{1 g}$ mode is too low in energy to avail itself of this extra decay path and thus exhibits no observable anomaly.

\subsection{Discussion of optical coupling coefficients}

From the general expression in Eq. (8) it is apparent that separating out the two spin-dependent contributions to the Raman intensity is not straightforward, as the relative weights of coefficients $B$ and $C$ are not known. For a partial analysis we may proceed as follows. In the limit of taking $T \rightarrow 0$ (which implies $\Phi \rightarrow 1$ ) in Eq. (9), which is valid for all $T<T_{N}$, we have

$$
I(0)=\left|A-B S^{2}\right|^{2}+C^{2} S^{2}, \quad T=0 .
$$

In the absence of any spin-phonon coupling the result would simply be

$$
I_{0}(0)=|A|^{2}, \quad T=0
$$


We obtain a third relationship by considering the form taken by Eq. (9) when $T=T_{N}$. Since $\Phi$ is given by Eq. (6) in this case and $\left\langle S^{Z}>=0\right.$ we have

$$
I\left(T_{N}\right)=\left(n_{\mathrm{ph}}+1\right)\left|A-\frac{1}{7} S(S+1) B\right|^{2}, T=T_{N} .
$$

Here it is understood that the Bose-Einstein thermal population factor $n_{\mathrm{ph}}$ is evaluated with the relevant phonon frequency at $T=T_{N}$.

Of the four Raman-active vibrational modes, only the $A_{1 g}$ and $E_{g}$ modes exhibit a strong variation with temperature below $T_{N}$ and thus we choose these two modes for further analysis here. In both cases, the mode intensity for $\mathrm{MnF}_{2}, \mathrm{FeF}_{2}$, and $\mathrm{NiF}_{2}$ increases with decreasing temperature below $T_{N}$, while for $\mathrm{CoF}_{2}$ it decreases in intensity. On applying the same general procedure as used for the mode frequencies, but in this case utilizing Eqs. (10)-(12), we find for each compound the approximate relative values of the $A, B$, and $C$ coefficients with the numerical results for $|B / A|$ and $|C / A|$ being presented in Table 3.

Table 3. Deduced values $|B / A|$ and $|C / A|$ for the ratio of intensity coefficients of the $A_{1 g}$ and $E_{g}$ modes for the four compounds. In the case of the $A_{1 g}$ mode for $\mathrm{FeF}_{2}$ and $\mathrm{CoF}_{2}$ the coefficients are obtained separately for the $(X X)$ and $(Z Z)$ polarizations; otherwise the polarizations are as indicated earlier in the text

\begin{tabular}{c|c|c|c|c}
\hline \hline \multirow{2}{*}{ Compound } & \multicolumn{2}{|c|}{$A_{1 g}$ mode } & \multicolumn{2}{c}{$E_{g}$ mode } \\
\cline { 2 - 5 } & $|B / A|$ & $|C / A|$ & $|B / A|$ & $|C / A|$ \\
\hline \hline $\mathrm{MnF}_{2}$ & $<0.1$ & 0.3 & $<0.1$ & 0.3 \\
$\mathrm{FeF}_{2}(X X)$ & 0.1 & 0.5 & $<0.1$ & 0.7 \\
$\mathrm{FeF}_{2}(\mathrm{ZZ})$ & $<0.1$ & 0.5 & & \\
$\mathrm{NiF}_{2}$ & 0.1 & 0.8 & 0.2 & 0.8 \\
$\mathrm{CoF}_{2}(X X)$ & 0.2 & 0.2 & 0.4 & 0.4 \\
$\mathrm{CoF}_{2}(\mathrm{ZZ})$ & 0.3 & 0.3 & & \\
\hline \hline
\end{tabular}

These approximate results (which are estimated to be reliable to \pm 0.1 ) allow us to make several general observations regarding the magnitudes of the coefficients and (in some cases) their signs. First, we see that $|B / A|$ is generally smaller than $|C / A|$ for $\mathrm{MnF}_{2}, \mathrm{FeF}_{2}$, and $\mathrm{NiF}_{2}$. This behavior indicates that the stronger effect of the spin-phonon coupling comes via the $\left\langle\mathbf{S}_{i} \cdot \mathbf{S}_{j}>\right.$ term in Eq. (8). By contrast, the $B$ and $C$ coefficients for $\mathrm{CoF}_{2}$ are approximately equal for both modes. The reason for this different behavior for $\mathrm{CoF}_{2}$ remains an open question, but it may possibly be related to the strong spin-orbit and angular-momentum effects mentioned earlier, which are absent in the other compounds. Second, since the mode intensity increases below $T_{N}$ we can say that $A$ and $B$ have opposite signs for $\mathrm{MnF}_{2}, \mathrm{FeF}_{2}$, and $\mathrm{NiF}_{2}$, although the sign of $C$ remains uncertain. Third, the relative magnitudes of the coefficient ratios for each compound are remarkably similar for these two modes, including the relatively high value found for $|C / A|$ in $\mathrm{NiF}_{2}$.

\section{Conclusions}

A comprehensive theoretical analysis of the spinphonon coupling in rutile-structure antiferromagnets has allowed a detailed interpretation of existing experimental work on the four Raman-active phonons found in $\mathrm{MnF}_{2}$, $\mathrm{FeF}_{2}$, and $\mathrm{NiF}_{2}$ and new experimental work on $\mathrm{CoF}_{2}$. Although the absolute value for the spin-phonon coupling coefficients can have a wide range of values between phonons and as well as between compounds, it is discovered that the spin value has a prominent scaling effect that, when taken into account, results in parameter values that are much closer together. From an analysis of the variation in temperature of the $A_{1 g}$ and $E_{g}$ phonon Raman intensities, it is revealed that $\mathrm{MnF}_{2}, \mathrm{FeF}_{2}$, and $\mathrm{NiF}_{2}$ have very similar behaviors for the ratios $|B / A|$ and $|C / A|$ of the magneto-optic coefficients, while in $\mathrm{CoF}_{2}$ these ratios are approximately equal for both phonon modes. The theoretical approach developed here can be applied to most antiferromagnets and it would be informative to investigate the phonon behavior below the magnetic ordering temperature in other series of antiferromagnetic systems to see if the results obtained here for rutile structure compounds are universal.

\section{Acknowledgments}

This paper is dedicated to the memory of Professor Victor V. Eremenko (1932-2016), formerly of the B. Verkin Institute of Low Temperature Physics and Engineering and Editor in Chief of this journal (Low Temperature Physics), whose pioneering work on the measurement and comprehension of the optical properties of magnetic systems continues to be of great value to us all. MGC gratefully acknowledges partial support from the Natural Sciences and Engineering Research Council (NSERC) of Canada through Discovery Grant RGPIN-2017-04429. We thank H.J. Labbé for the crystal sample preparation and R. Radomski and J. Johnson for curve fitting the $\mathrm{CoF}_{2}$ Raman spectra.

1. M.G. Cottam and D.J. Lockwood, Light Scattering in Magnetic Solids, Wiley, New York (1986).

2. D.J. Lockwood, in Light Scattering in Solids III, M. Cardona and G. Güntherodt (eds.), Springer, Heidelberg (1982).

3. D.J. Lockwood, in Magnetic Excitations and Fluctuations, S.W. Lovesey, U. Balucani, F. Borsa, and V. Tognetti (eds.), Springer, Berlin (1984), p. 59.

4. I.W. Johnstone, D.J. Lockwood, G. Mischler, J.R. Fletcher, and C.A. Bates, J. Phys. C 11, 4425 (1978).

5. For details of the spin-phonon coupling in the Eu and Cd$\mathrm{Cr}$ compounds, see the review by G. Guntherodt and R. Zeyher, in Light Scattering in Solids IV, M. Cardona and G. Guntherodt (eds.), Springer, Heidelberg (1984).

6. A. Akhiezer, Fiz. Zh. 10, 217 (1946).

7. E. Pytte, Ann. Phys. (NY) 32, 377 (1965). 
8. M.J. Jones and M.G. Cottam, Phys. Status Solidi B 66, 651 (1974).

9. M.J. Jones and M.G. Cottam, Phys. Status Solidi B 67, 75 (1975).

10. M.G. Cottam, J. Phys. C 7, 2901 (1974).

11. M.G. Cottam, J. Phys. C 7, 2919 (1974).

12. D.J. Lockwood and M.G. Cottam, in Magnetic Excitations and Fluctuations II, U. Balucani, S.W. Lovesey, M.G. Rasetti, and V. Tognetti (eds.), Springer, Berlin (1987), p. 186.

13. D.J. Lockwood and M.G. Cottam, J. Appl. Phys. 64, 5876 (1988).

14. E.A. Turov and Yu.P. Irkhin, Fiz. Met. Metalloved. 3, 15 (1956).

15. C. Kittel, Phys. Rev. 110, 836 (1958).

16. J.L. Sauvajol, R. Almairac, C. Benoit, and A.M. Bon, in Lattice Dynamics, M. Balkanski (ed.), Flammarion, Paris (1978), p. 199.

17. D.J. Lockwood, R.S. Katiyar, and V.C.Y. So, Phys. Rev. B 28, 1983 (1983).

18. D.J. Lockwood, in: Proc. IXth Int. Conf. on Raman Spectroscopy, Chem. Soc. Jpn., M. Tsuboi (ed.), Tokyo (1984), p. 810.

19. D.J. Lockwood, Fiz. Niz. Temp. 28, 709 (2002) [Low Temp. Phys. 28, 505 (2002)].

20. C. Binek and W. Kleeman, J. Phys. C 4, 65 (1992).

21. R.M. Macfarlane and S. Ushioda, Solid State Commun. 8, 1081 (1970).

22. R.M. Macfarlane and H. Morawitz, Phys. Rev. Lett. 27, (1971).

23. E. Meloche, M.G. Cottam, and D.J. Lockwood, Fiz. Niz. Temp. 40, 173 (2014) [Low Temp. Phys. 40, 134 (2014)].

24. E. Meloche, M.G. Cottam, and D.J. Lockwood, Phys. Rev. B 76, 104406 (2007).

25. L.J. Zimring and J.W. Stout, J. Chem. Phys. 51, 4197 (1969).

26. D.J. Lockwood and Z.R. Wasilewski, Phys. Rev. B 70, 155202 (2004).

27. A. Perakis, E. Sarantopoulou, Y.S. Raptis, and C. Raptis, Phys. Rev. B 59, 775 (1999).

28. E.F. da Silva, Jr., F.L.A. Machado, and S.M. Rezende, Solid State Commun. 48, 1077 (1983).

29. R.S. Katiyar, J. Phys. C 3, 1087 and 1693 (1970).

30. J.S. Smart, Effective Field Theories of Magnetism, Saunders, Philadelphia (1966).

31. R.J. Elliott, J. Phys. Chem. Solids 16, 165 (1960).

32. W. Hayes and R. Loudon, Scattering of Light by Crystals, Wiley, New York (1978).

33. E.F. Steigmeier and G. Harbeke, Phys. Kondens. Materie 12, 1 (1970).
34. K. Wakamura, Solid State Commun. 71, 1033 (1989).

35. M.T. Hutchings, M.F. Thorpe, R.J. Birgeneau, P.A. Fleury, and H.J. Guggenheim, Phys. Rev. B 2, 1362 (1970).

\section{Спін-фрононна взаємодія в діфрлуоридах перехідних металів: теорія та експеримент}

\section{M.G. Cottam, D.J. Lockwood}

3 використанням останніх результатів експериментально зі застосуванням спектроскопії непружного розсіяння світла та теоретично методом модифікованого середньопольового підходу для оцінки кореляційних функцій спінової пари проведено повне порівняльне дослідження спін-фононних взаємодій в декількох діфлуоридах перехідних металів рутилової структури, зокрема $\mathrm{FeF}_{2}, \mathrm{MnF}_{2}, \mathrm{NiF}_{2}$ та $\mathrm{CoF}_{2}$. 3 метою отримання оцінок коефіцієнтів спін-фононного зв'язку та відносних величин коефіцієнтів магнітооптичного зв'язку представлено нові експериментальні данні, які інтерпретуються в рамках всебічного розширеного теоретичного опису.

Ключові слова: антиферомагнетики, спін-фононний зв'язок, структура рутилу, магнітооптичний зв'язок, раманівська спектроскопія.

\section{Спин-фононное взаимодействие в дифрлуоридах переходных металлов: теория и експеримент}

\section{M.G. Cottam, D.J. Lockwood}

С использованием последних результатов экспериментально с применением спектроскопии неупругого рассеяния света и теоретически методом модифицированного среднеполевого подхода для оценки корреляционных функций спиновой пары проведено полное сравнительное исследование спин-фононных взаимодействий в нескольких дифлуоридах переходных металлов рутиловой структуры, в частности $\mathrm{FeF}_{2}$, $\mathrm{MnF}_{2}, \mathrm{NiF}_{2}$ и $\mathrm{CoF}_{2}$. С целью получения оценок коэффициентов спин-фононной связи и относительных величин коэффициентов магнитооптической связи представлены новые экспериментальные данные, которые интерпретируются в рамках всестороннего расширенного теоретического описания.

Ключевые слова: антиферромагнетики, спин-фононная связь, структура рутила, магнитооптическая связь, рамановская спектроскопия. 